\title{
Degradable PHBHHx Modified by the Silk Fibroin for the Applications of Cardiovascular Tissue Engineering
}

\author{
Hua-Xiao Yang, Min Sun, Yi Zhang, and Ping Zhou \\ The Key Laboratory of Molecular Engineering of Polymers, Ministry of Education, Department of Macromolecular Science, \\ Fudan University, Shanghai 200433, China \\ Correspondence should be addressed to Ping Zhou, pingzhou@fudan.edu.cn
}

Received 19 August 2011; Accepted 18 September 2011

Academic Editor: J. Y. Lu

Copyright (๑) 2011 Hua-Xiao Yang et al. This is an open access article distributed under the Creative Commons Attribution License, which permits unrestricted use, distribution, and reproduction in any medium, provided the original work is properly cited.

Biodegradable polymer, poly (3-hydroxybutyrate-co-3-hydroxyhexanoate) (PHBHHx), has been used to fabricate the tissueengineered cardiovascular scaffolds due to its controllable and suitable mechanical properties. However, the unsound biocompatibility of PHBHHx is still the unsolved problem till now. Silk fibroin (SF) with low inflammation, no blood clotting, and good cell and tissue compatibility in vitro and vivo is adopted as a surface modificator to improve the biocompatibility of PHBHHx. The adhesion of SF on PHBHHx surface was investigated by infrared spectroscopy and water contact angle. The proliferation and morphologies of human fibroblasts, human smooth muscle cells, endothelial-like cell line ECV304, and umbilical vein endothelial cells on the SF-modified PHBHHx were studied by the assays of MTT, H\&E staining, DAPI staining, and the scanning electron microscopy. In sum, SF-modified PHBHHx scaffolds are highly biocompatible with cardiovascular-related cells, demonstrating its potential help for the extensive applications of PHBHHx in the cardiovascular regeneration.

\section{Introduction}

In the field of cardiovascular disease, more than 100,000 patients in USA every year need to have their dysfunctional or diseased valves replaced with the prosthetic valves [1]. The traditional artificial heart valves are made from inert metals or nondegradable polymers to maintain the basic physiological functions. These types of artificial heart valves have the tendency to produce thrombus and have to be renewed several years after implantation, therefore, cannot be regarded as an ultimate replacement for patients, especially for infants or children. Considering the patient compliance, many researchers have attempted to find an alternate material that is capable of biomimicking the extracellular matrix (ECM) for cell attaching, proliferating, differentiating, and immigrating [2].

Biodegradable polymers, such as poly(L-lactide-co-glycolide) (PLGA), poly( $\mathcal{E}$-caprolactone) (PCL), and polyhydroxyalkanoates (PHAs), have been widely investigated during recent decades [3-6] for the tissue-engineering application. Among these polymers, PHAs family (such as PHB, PHBV) is well known for its low toxicity as well as therapeutic or nutritional benefits to cells [7]. In addition, as a degradation product from PHAs, hydroxyl butyrate (HB) is a normal metabolized component in the blood circulation system. It has also been found that HB can promote the L929 cells proliferating in vitro [8]. Among PHAs family, PHBHHx is a new member, copolymer of hydroxyl butyrate (HB), and hydroxyl hexanoate $(\mathrm{HH})$, which is synthesized by the microorganism [7]. The " $x$ " in PHBHHx represents the content of hydroxyl hexanoate. The proper content of hydroxyl hexanoate ensures PHBHHx have low crystallinity, resulting in a higher tensile strength and strain of PHBHHx than that of PHB $[3,9,10]$, therefore, the adjustable molecular weight and composition ratio enable the PHBHHx to meet the requirements of various tissue-engineered organs [9], such as tissue-engineered heart valve [11] and bone [12]. Therefore, PHBHHx has become one of promising candidates for tissue-engineering material. Qu et al. transplanted the PHBHHx, PLA, and PHB films into the back of New Zealand white rabbits. It was found that PHBHHx showed a very mild tissue response during 6-month test, compared to other two films [13]. However, many biodegradable polymers usually have high hydrophobicity and lack of the recognition domains for the cells. Therefore, people have tried to improve the polymer hydrophilicity by various 
methods. Ho et al. mobilized the RGD peptides on the PLLA membrane to improve its biocompatibility [14]. To improve the affinity of fibroblasts, Wang et al. introduced the functional hydrophilic chemical groups, such as $-\mathrm{NH}_{2}$ and $-\mathrm{OH}$, by treating the polymer surface with plasma technique. Silk fibroin (SF) from cocoon of silkworm has been demonstrated to have the ability of anti-blood clotting [15] and less inflammation reactions to the macrophage [16], therefore, it has been attended recently. During past years, natural silk fibroin has been used in our laboratory to modify the surface of PHBHHx (HHx $=12 \mathrm{~mol} \%)$ and attempted to make the cardiovascular cells, fibroblasts, smooth muscle cells (SMCs), and endothelial cells (ECs) proliferate well on the polymer surface $[6,17-19]$. We have demonstrated that PHBHHx scaffolds modified by the silk fibroin not only have the good mechanical properties of the synthetic polymer, but also have the excellent biocompatibility of the natural biomacromolecules. In this review, we would like to introduce some of research results about our work.

\section{Properties of PHBHHx}

2.1. Mechanic Property of PHBHHx. The cardiovascular tissue engineering requires the material having high tensile strength and strain to bear the dilatation. Therefore, it is important to have comparable mechanical properties of material with target tissue. Zhao et al. transplanted the PHB scaffold into a lamb for 20 weeks and found that the mechanical properties of the PHB-made new tissue resembled those of the native valve tissue [10]. The mechanical strength of PHBHHx (HHx $=12 \mathrm{~mol} \%)$ and silk fibroinmodified PHBHHx $(\mathrm{HHx}=12 \mathrm{~mol} \%)$ has been investigated in the previous work in our laboratory [20]. The results are shown in Figure 1. It can be concluded from Figure 1 that the stress-stain curve of SF-modified PHBHHx (Figure 1(a)) and PHBHHx (Figure 1(b)) films is in similar shape, which is the typical pattern of the plastic material. The SF-modified PHBHHx films have the maximum tensile strength of $11.5 \pm$ $0.5 \mathrm{MPa}$ and the elongation at break of $175 \pm 5 \%$, slightly lower than that of PHBHHx film, which is $11.7 \pm 0.5 \mathrm{MPa}$ and $204 \pm 5 \%$, respectively. The small decrease in the elongation of SF-modified PHBHHx film might result from the addition of silk fibroin in the PHBHHx film which is swelled by the water/acetone solution during the process of modification [20]. The native cardiovascular tissue has yield strength of about $0.7 \sim 8 \mathrm{MPa}$ and a maximum elongation of $150 \%$ [10]. Therefore, SF-modified PHBHHx material is a potential material applicable in the cardiovascular tissue engineering because of its proper mechanical properties.

2.2. Water Diffusion in PHBHHx. When biodegradable PHBHHx is used to construct a scaffold and implanted into the body, nutrition transportation is an important issue. Water diffusion ability in the scaffold plays a key role in the transportation process. Water diffusion is affected by many factors, such as polymer categories, crystalline degree. We used time-resolved attenuated total reflection Fourier transform infrared spectroscopy (ATR-FTIR) to investigate

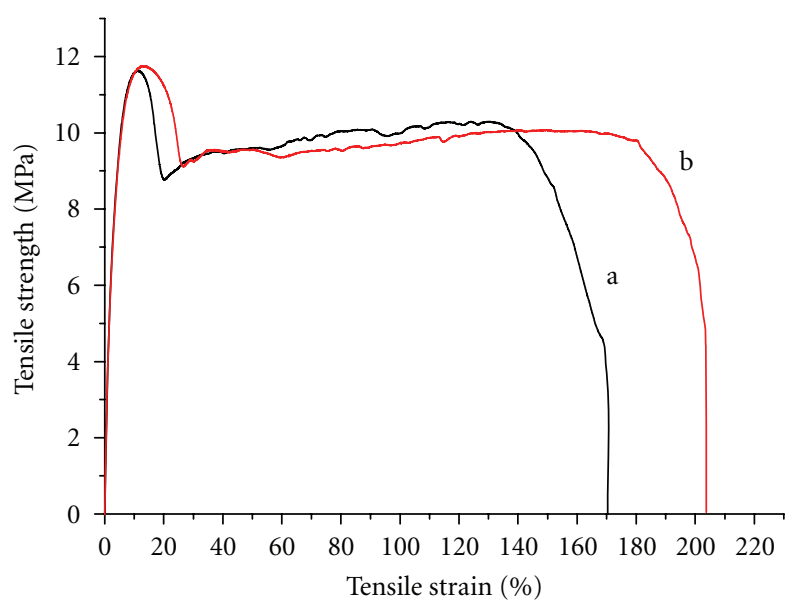

Figure 1: Stress-strain curves of SF/PHBHHx films (a) and PHBHHx films (b) with a constant elongation rate of $5 \mathrm{~mm} / \mathrm{min}$. Four samples in the shape of rectangle in length $\times$ width $\times$ thickness of $40 \times 5 \times 0.1 \mathrm{~mm}$ were tested for each film [20].

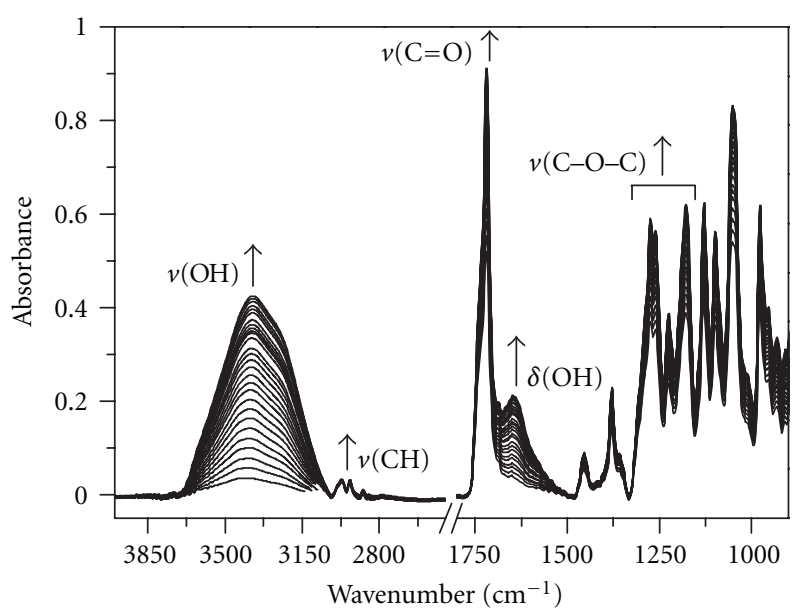

FIgURE 2: ATR-FTIR spectra of PHBHHx ( $\mathrm{HHx}=12 \mathrm{~mol} \%)$ as water diffusion. The arrows indicate the directions of intensity changes during diffusion process [21].

the process of water diffusion in the PHBHHx $(\mathrm{HHx}=$ 12 mol\%) matrix with crystalline degree of $16.2 \pm 0.3 \%$ [21].

Time-resolved ATR-FTIR is a powerful technique for the measurement of small molecule diffusion in situ in the polymer [21]. Figure 2 is ATR-FTIR spectra of the PHBHHx film as water diffusion and shows the increased absorbance during water diffusion. Here, the stretching and bending bands of $-\mathrm{OH}$ of water molecules are utilized to evaluate the water diffusion coefficient. The experimental data from the integrals of $v(\mathrm{OH})$ stretching band at $3700 \sim 3000 \mathrm{~cm}^{-1}$ (Figure 3(a)) and $\delta(\mathrm{OH})$ bending band at $1700 \sim 1550 \mathrm{~cm}^{-1}$ (Figure 3(b)) versus diffusion time and the exponentially stimulated curves are shown in Figure 3. Two parallel samples, Sample I and II, of the PHBHHx films were measured for the average result.

From Figure 3, the water diffusion coefficient of $7.8 \pm$ $0.7 \times 10^{-8} \mathrm{~cm}^{2} \mathrm{~s}^{-1}$ in the PHBHHx is deduced. Compared 


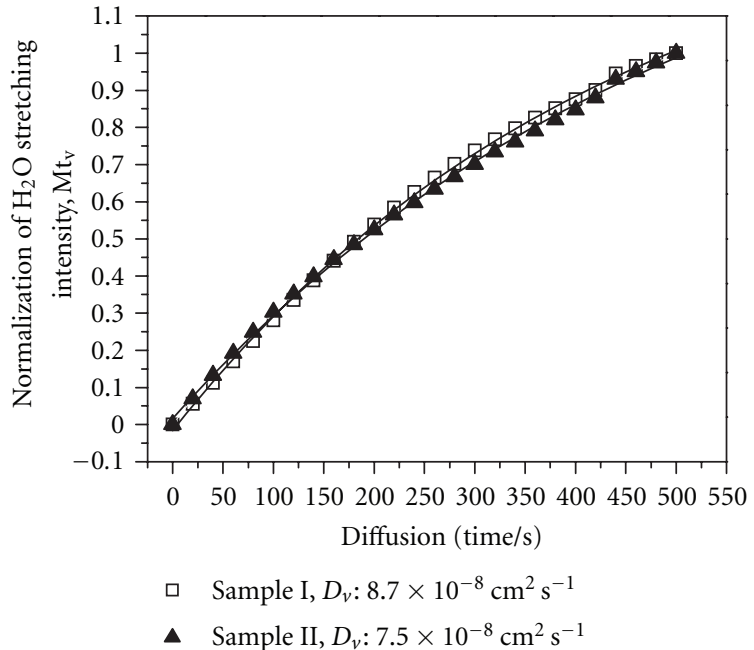

(a)

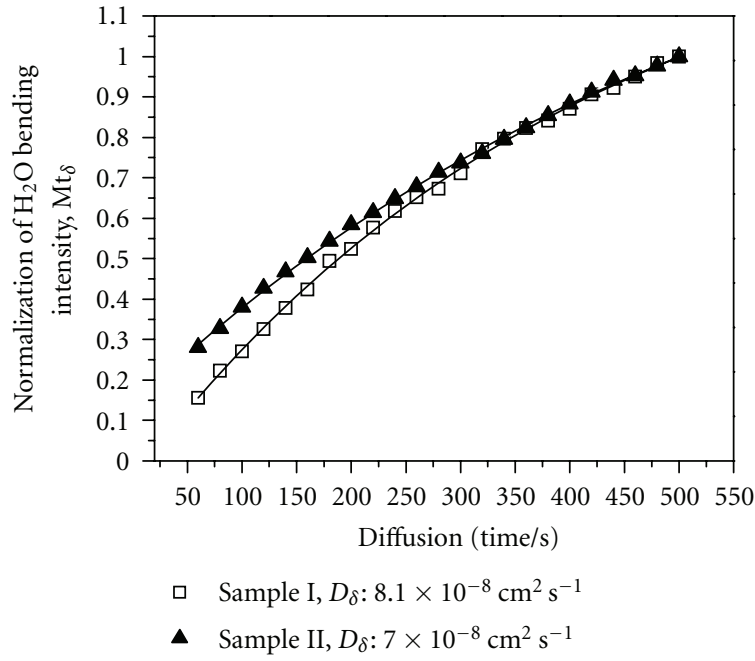

(b)

Figure 3: Diffusion curve of water in PHBHHx $(\mathrm{HHx}=12 \mathrm{~mol} \%)$ matrix with crystalline degree of $16.2 \pm 0.3 \%$. The hollow square and solid triangle points represent experimental data of Sample I and II, respectively, and the solid lines represent fitted curves based on Fickian Model. The thickness of the polymer film $L=97 \pm 1 \mu \mathrm{m}$. (a) represents $v(\mathrm{OH})$ stretching band at $3700 \sim 3000 \mathrm{~cm}^{-1}$ versus diffusion time; (b) represents $\delta(\mathrm{OH})$ bending band at $1700 \sim 1550 \mathrm{~cm}^{-1}$ versus diffusion time. The diffusion coefficient $D_{v}$ derived from $v(\mathrm{OH})$ stretching band is $8.7 \times 10^{-8} \mathrm{~cm}^{2} \mathrm{~s}^{-1}$ (Sample I, $\left.R_{v}^{2}=0.9994\right)$ and $7.5 \times 10^{-8} \mathrm{~cm}^{2} \mathrm{~s}^{-1}$ (Sample II, $\left.R_{v}^{2}=0.9990\right)$ and $D_{\delta}$ derived from $\delta(\mathrm{OH})$ bending band is $8.1 \times 10^{-8} \mathrm{~cm}^{2} \mathrm{~s}^{-1}$ (Sample $\mathbf{I}, R_{\delta}^{2}=0.9990$ ) and $7.0 \times 10^{-8} \mathrm{~cm}^{2} \mathrm{~s}^{-1}$ (Sample II, $R_{\delta}^{2}=0.9992$ ) at $293 \mathrm{~K}$. The average water diffusion coefficient $D$ and standard deviation of $7.8 \pm 0.7 \times 10^{-8} \mathrm{~cm}^{2} \mathrm{~s}^{-1}$ was determined by two pairs of $D_{v}$ and $D_{\delta}$ [22].

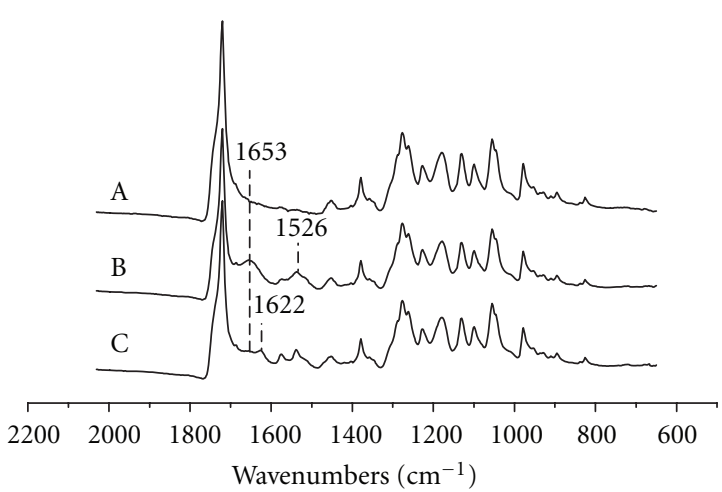

Figure 4: ATR-FTIR spectra of the surfaces of (A) PHBHHx 3D scaffold; (B) the SF-modified PHBHHx 3D scaffold without methanol treatment; (C) the same scaffold as (B) but with methanol treatment [19].

with the water diffusion coefficient of $1.5 \times 10^{-8} \mathrm{~cm}^{2} \mathrm{~s}^{-1}$ in PHB $[22,23]$, the larger water diffusion coefficient of PHBHHx than that of PHB indicates that PHBHHx is more to the tissue-engineering materials, especially to the transportation of water and the water-soluble nutrition into the cells through the polymer scaffold.

\section{Properties of Silk Fibroin on the PHBHHx Surface}

3.1. Adhesion Ability of Silk Fibroin on the PHBHHx Surface. $0.75 \mathrm{wt} \%$ silk fibroin solution was used to modify the porous

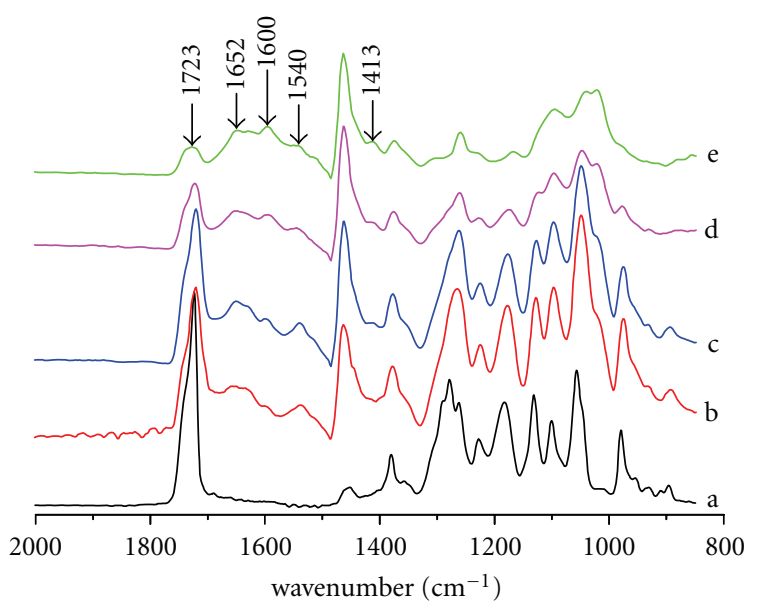

Figure 5: ATR-FTIR spectra of PHBHHx and SF/PHBHHx porous scaffolds. (a) PHBHHx porous scaffold; (b)-(e) SF-modified PHB$\mathrm{HHx}$ porous scaffolds flushed with $\mathrm{PBS}$ solution $(\mathrm{pH}=7.4)$ at $37^{\circ} \mathrm{C}$ for $0,7,14$, and 21 days, respectively. The flush rate was $55 \mathrm{~mL} / \mathrm{min}$ which was the blood impulse rate under normal physiological condition [20].

PHBHHx scaffold $[19,20]$. The results were characterized by ATR-FTIR which can noninvasively detect the chemical groups on the surface of the film.

ATR-FTIR analysis is performed to examine the presence of the silk fibroin on the surface of SF-modified PHBHHx porous scaffold as well as the behavior of silk fibroin conformation. The bands of Amide I and Amide II in infrared (IR) spectrum are very useful for the conformational 


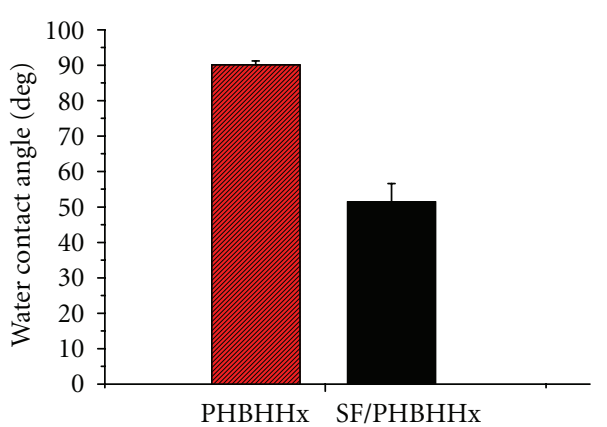

Figure 6: Static water contact angles of PHBHHx and SF-modified PHBHHx films [20, 28].

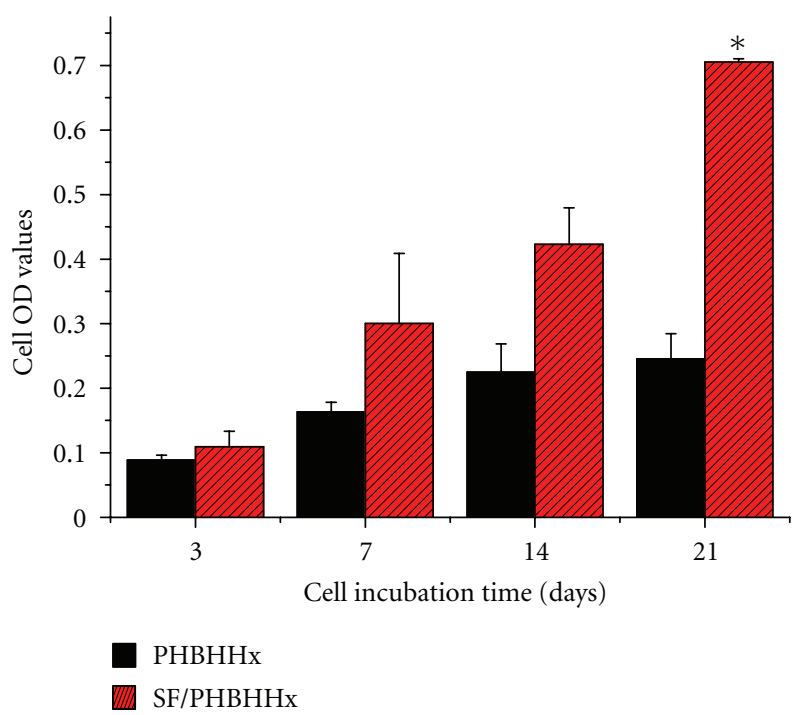

FIGURE 7: OD values of the human fibroblasts on the SF-modified porous $\mathrm{PHBHHx}$ scaffolds at different culture times, with porous PHBHHx scaffold as control: PHBHHx with pore size between 50 $75 \mu \mathrm{m} .0 .75 \%$ silk fibroin was used for the modification. Error bars represent means $\pm \mathrm{SD}$ for $n=4 .{ }^{*} P<0.05$ [19].

analysis of the protein. In general, the Amide I mode of silk fibroin associating with the $\alpha$-form conformation appears at $1650-1660 \mathrm{~cm}^{-1}$, whereas the random coil conformation at $1640-1650 \mathrm{~cm}^{-1}$ and the $\beta$-form conformation at 1620 and $1640 \mathrm{~cm}^{-1}$; the Amide II mode of silk fibroin appear at 1520 $1530 \mathrm{~cm}^{-1}$.

Figure 4 represents the ATR-FTIR spectra of $\mathrm{PHBHHx}$ porous scaffold (Figure 4(A)), SF-modified scaffold without (Figure 4(B)) and with (Figure 4(C)) methanol treatment, respectively. It can be observed that there are some peculiar peaks, 1653, 1622, and $1526 \mathrm{~cm}^{-1}$ appear in both spectra of Figures 4(B) and 4(C) when compared with Figure 4(A), which demonstrates the presence of silk fibroin on the surfaces of SF-modified PHBHHx scaffolds as have been proved in a film by Cai et al. [24]. Notably, the peak at $1653 \mathrm{~cm}^{-1}$ (Figure 4(B)) indicates that the silk fibroin conformation is $\alpha$-form before the scaffold is treated by methanol. However, the silk fibroin conformation transition from water-soluble $\alpha$-form to water-insoluble $\beta$-form happens after methanol
TABle 1: Assignments of FTIR spectra of PHBHHx and SF/ PHBHHx samples.

\begin{tabular}{|c|c|c|}
\hline Peaks $\left(\mathrm{cm}^{-1}\right)$ & Assignments & References \\
\hline 1652 & Amide I of silk fibroin & \multirow{2}{*}{ [20] } \\
\hline 1540 & Amide II of silk fibroin & \\
\hline 1723 & $\begin{array}{l}\mathrm{C}=\mathrm{O} \text { stretching band in } \\
\mathrm{PHBHHx}\end{array}$ & \multirow{4}{*}[22,25]{} \\
\hline 1600 & $\begin{array}{l}\text { symmetric stretching band of } \\
\mathrm{COO}^{-} \text {in } \mathrm{PHBHHx}\end{array}$ & \\
\hline 1460 & $\begin{array}{l}\mathrm{CH}_{3} \text { asymmetric bending band } \\
\text { in } \mathrm{PHBHHx}\end{array}$ & \\
\hline 1413 & $\begin{array}{l}\text { asymmetric stretching band of } \\
\mathrm{COO}^{-} \text {in } \mathrm{PHBHHx}\end{array}$ & \\
\hline 1230 & $\begin{array}{l}\mathrm{C}-\mathrm{O}-\mathrm{C} \text { stretching band in } \\
\mathrm{PHBHHx}\end{array}$ & \\
\hline
\end{tabular}

treatment, which is evidenced by the Amide I peak shifted from $1653 \mathrm{~cm}^{-1}$ (Figure 4(B)) to $1622 \mathrm{~cm}^{-1}$ (Figure 4(C)), resulting in the immobilization of the silk fibroin on the scaffolds [17]. Therefore, to prevent the dissolution of the coated silk fibroin from the scaffolds in our experimental conditions, all the SF-modified scaffolds are treated by methanol.

If the SF-modified PHBHHx scaffold were applied into the cardiovascular tissue engineering, it would bear the high sheer-stress of blood flow in the blood vessel, so a firm adhesion of SF on the surface of scaffold is required. We tested the ability of silk fibroin adhered on the $\mathrm{PHBHHx}$ surface under a physiologically flushed condition by phosphate buffer saline (PBS, pH = 7.4) solution. Here, ATR-FTIR was used to detect the presence of silk fibroin on the surface of SFmodified PHBHHx porous scaffold before and after flushed with PBS solution for different periods of flushed time. Since the low concentration of SF solution could penetrate through the SF-modified PHBHHx scaffold, it was considered that the SF could be absorbed not only on the surface of porous scaffold, but also in the scaffold pores. Figure 5(a) is the ATRFTIR spectrum of PHBHHx scaffold and Figures 5(b)-5(e) show the spectra of SF-modified PHBHHx scaffolds flushed for $0,7,14$, and 21 days, respectively. The peak assignments of FTIR spectrum of PHBHHx and SF/PHBHHx samples are summarized in Table 1.

Compared with the peaks in the spectrum of $\mathrm{PHBHHx}$ scaffold (Figure 5(a)), the peaks at $1652 \mathrm{~cm}^{-1}$ and $1540 \mathrm{~cm}^{-1}$ from silk fibroin $[19,25]$ are found in the spectra of SFmodified PHBHHx (SF/PHBHHx) scaffolds (Figures 5(b)5(e)). It demonstrates that silk fibroin was successfully absorbed on the porous scaffold, even the scaffolds were flushed by PBS solution for 21 days. It was also found that for the PHBHHx scaffold, the $\mathrm{C}=\mathrm{O}$ peak at $1723 \mathrm{~cm}^{-1}$ decreases gradually while the new peaks at $1600 \mathrm{~cm}^{-1}$ and $1413 \mathrm{~cm}^{-1}$ increase after being flushed for 21 days, demonstrating the partial hydrolysis of the PHBHHx. Meanwhile, the peak at $1230 \mathrm{~cm}^{-1}$ decreases, which indicates the helical conformation of PHBHHx changes [26]. The intensity of peak at $1460 \mathrm{~cm}^{-1}$ in $\mathrm{PHBHHx}$ scaffold slightly increases during 


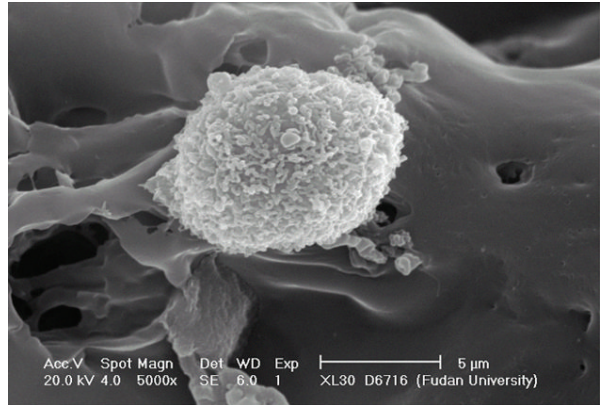

(a)

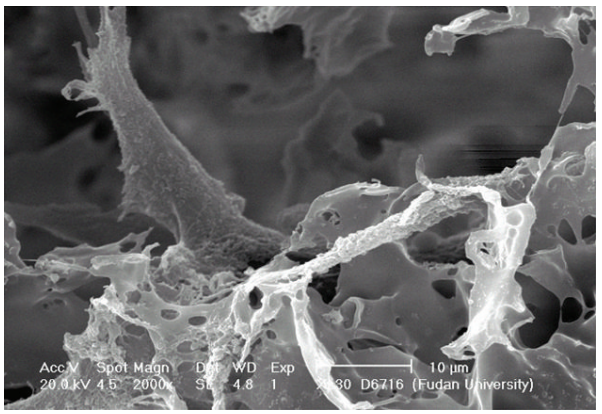

(c)

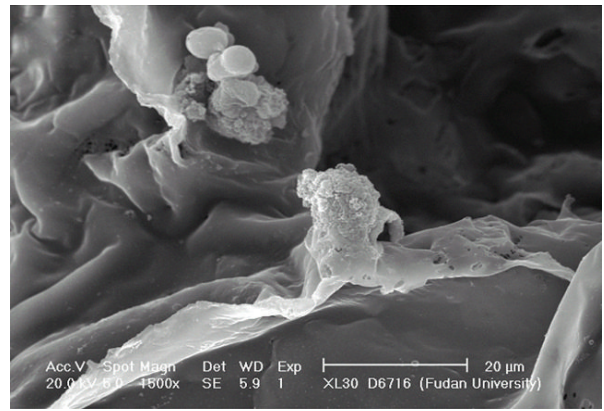

(b)

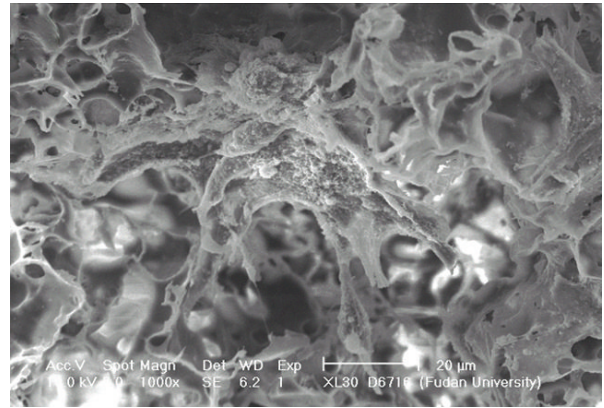

(d)

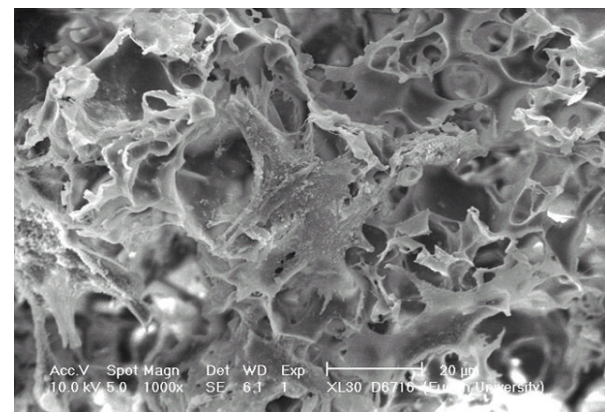

(e)

FIgURE 8: Representative SEM images of the cells cultured on the porous PHBHHx scaffolds: (a) and (b) are the cells attached on the PHBHHx and SF-modified scaffolds for 1 day, respectively; (c) are the cells spreading in the pores of SF-modified scaffolds in 3 days of culture; (d) and (e) are the cell confluence on the surface and in the pores of SF-modified scaffolds, respectively, in 7 days of culture [19].

flushing process, indicating the presence of hydrolyzed products in the scaffold. The repeated experiments herein prove that the peak at $1460 \mathrm{~cm}^{-1}$ is much stronger in the flushed SF/PHBHHx scaffold than that in the SF/PHBHHx scaffold, perhaps due to $\mathrm{PBS}$ solution changing the polar environment of $\mathrm{CH}_{3}$ group [26]. Conclusively, we think that the silk fibroin anchors on the scaffold by the hydrogen bonding interaction with PHBHHx [19], even when the PHBHHx is partially hydrolyzed in the mimic flowing blood system.

\subsection{Hydrophilic Property of Silk Fibroin-Modified PHBHHx.} The water contact angle of material surface can be used to characterize the hydrophilicity of SF-modified PHBHHx. It is found in Figure 6 that the static water contact angle of PHBHHx film decreases from $90^{\circ}$ to $52^{\circ}$ after PHBHHx film is modified by silk fibroin, which demonstrates that the silk fibroin considerably affects the surface wettability of the scaffold, making the scaffold more hydrophilic.

Moreover, based on the measurement of $\mathrm{H}_{2} \mathrm{O}$ and $\mathrm{CH}_{2} \mathrm{I}_{2}$ contact angles on the material surface, the surface free energy of PHBHHx and SF-modified PHBHHx can be calculated according to the following equations:

$$
\begin{aligned}
& \left(1+\cos \theta_{1}\right) \gamma_{1}=4\left(\frac{\gamma_{1}^{d} \gamma_{s}^{d}}{\gamma_{1}^{d}+\gamma_{s}^{d}}+\frac{\gamma_{1}^{p} \gamma_{s}^{p}}{\gamma_{1}^{p}+\gamma_{s}^{p}}\right), \\
& \left(1+\cos \theta_{2}\right) \gamma_{2}=4\left(\frac{\gamma_{2}^{d} \gamma_{s}^{d}}{\gamma_{2}^{d}+\gamma_{s}^{d}}+\frac{\gamma_{2}^{p} \gamma_{s}^{p}}{\gamma_{2}^{p}+\gamma_{s}^{p}}\right),
\end{aligned}
$$

where $\gamma_{1}$ and $\gamma_{2}$ are the total surface energies of $\mathrm{H}_{2} \mathrm{O}$ and $\mathrm{CH}_{2} \mathrm{I}_{2}$ on the material surface, respectively; $\gamma^{d}$ represents the dispersive components, $\gamma^{p}$ represents the polar components; $\theta_{1}$ is contact angle of $\mathrm{H}_{2} \mathrm{O} ; \theta_{2}$ is contact angle of $\mathrm{CH}_{2} \mathrm{I}_{2}$. For 


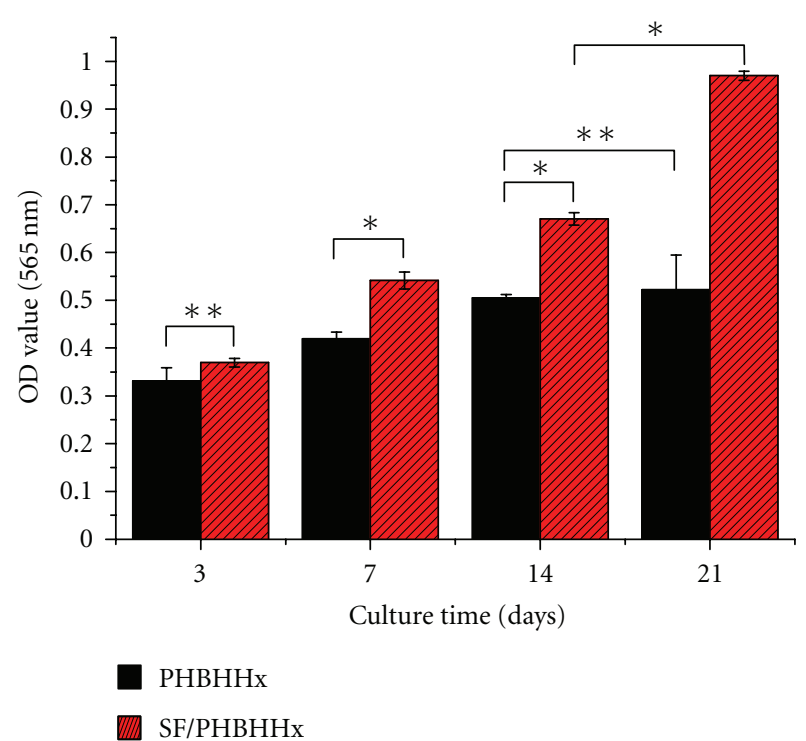

Figure 9: The optical density value of the hSMCs on the SF-modified PHBHHx scaffold with PHBHHx scaffold as control. $n=3, * P<0.01$, ${ }^{* *} P>0.05[25]$.

TABLE 2: The contact angle and surface free energy of SF-modified PHBHHx and PHBHHx films [28].

\begin{tabular}{lccccc}
\hline Sample & $\begin{array}{c}\theta_{\mathrm{H}_{2} \mathrm{O}} \\
\left({ }^{\circ}\right)\end{array}$ & $\begin{array}{c}\theta_{\mathrm{CH}_{2} \mathrm{I}_{2}} \\
\left({ }^{\circ}\right)\end{array}$ & $\begin{array}{c}\gamma_{s}^{d} \\
\left(\mathrm{~mJ} / \mathrm{m}^{2}\right)\end{array}$ & $\begin{array}{c}\gamma_{s}^{p} \\
\left(\mathrm{~mJ} / \mathrm{m}^{2}\right)\end{array}$ & $\begin{array}{c}\gamma_{s}=\gamma_{s}^{d}+\gamma_{s}^{p} \\
\left(\mathrm{~mJ} / \mathrm{m}^{2}\right)\end{array}$ \\
\hline SF/PHBHHx & $52 \pm 5$ & $38 \pm 2$ & 40.5 & 16.9 & 57.4 \\
PHBHHx & $90 \pm 1$ & $46 \pm 1$ & 36.6 & 1.3 & 37.9 \\
\hline
\end{tabular}

$\mathrm{H}_{2} \mathrm{O}, \gamma_{1}=72.8 \mathrm{~mJ} / \mathrm{m}^{2}, \gamma_{1}^{d}=22.1 \mathrm{~mJ} / \mathrm{m}^{2}, \gamma_{1}^{p}=50.7 \mathrm{~mJ} / \mathrm{m}^{2}$. For $\mathrm{CH}_{2} \mathrm{I}_{2}, \quad \gamma_{2}=50.8 \mathrm{~mJ} / \mathrm{m}^{2}, \quad \gamma_{2}^{d}=44.1 \mathrm{~mJ} / \mathrm{m}^{2}, \quad \gamma_{2}^{p}=6.7 \mathrm{~mJ} / \mathrm{m}^{2}$. The total surface free energy $\left(\gamma_{s}\right)$ is sum of dispersive $\left(\gamma_{s}^{d}\right)$ and polar $\left(\gamma_{s}^{p}\right)$ components. The calculated surface free energies of PHBHHx and SF-modified PHBHHx are summarized in Table 2. It can be found that the $\gamma_{s}$ of SF-modified PHBHHx film is larger than that of unmodified PHBHHx film due to the larger polar component $\left(\gamma_{s}^{p}\right)$ of the SF-modified PHBHHx, demonstrating that the silk fibroin can make the PHBHHx more polar and hydrophilic.

As we know, cells favor the appropriately hydrophilic interface of scaffold for the growth and proliferation [27], the role of the silk fibroin does increase the hydrophilic property of the PHBHHx.

\section{Biocompatibility of Silk Fibroin-Modified PHBHHx}

The heart valve and blood vessel are composed by three important cells, fibroblasts, smooth muscle cells, and endothelial cells (ECs) as well [1] and [19, 25, 28]. The biocompatibility of human fibroblasts, human smooth muscle cells (hSMCs), and human umbilical vein endothelial cells (HUVECs) on the PHBHHx and SF-modified PHBHHx scaffolds were investigated.

\subsection{Human Fibroblasts on the Silk Fibroin-Modified PHBHHx}

4.1.1. Cells Vitality. The vitality of the fibroblasts cultured on the scaffolds is tested by MTT assay. MTT is the abbreviation of 3-(4,5-dimethylthiazol-2-yl)-diphenyltetrazolium bromide, which is a rapid colorimetric method to determine the viable cell numbers based on the mitochondrial conversion of the tetrazolium to MTT formazan. The higher optical density (OD) value of MTT indicates the more live cells on the scaffold. Figure 7 show the OD values of the fibroblasts cultured on the PHBHHx and SF-modified $\mathrm{PHBHHx}$ porous scaffolds for different days. It showed that the OD values are higher on the SF-modified PHBHHx scaffold than that on the unmodified ones cultured from 1 to 21 days.

4.1.2. Cells Morphology. The morphologies of the fibroblasts cultured on SF-modified PHBHHx porous scaffold in different culture days are observed by SEM images shown in Figure 8. It shows that the shapes of the cells on the unmodified (Figure 8(a)) and modified scaffolds (Figure 8(b)) look like sphere after one day of culture. After three days of culture, the cells elongate with shuttle-like shape (Figure 8(c)). After seven days of culture, the cells grow into confluence on the surface (Figure $8(\mathrm{~d})$ ) and in the pores (Figure $8(\mathrm{e})$ ) of SFmodified PHBHHx porous scaffolds.

As above, both of MTT assay and SEM observation prove the outstanding biocompatibility of the SF-modified PHBHHx porous scaffold for the fibroblasts cultured in vitro. Fibroblasts grow and proliferate better on modified scaffold than unmodified ones, and reach confluence on the surface of modified scaffold after 7 days of culture.

\subsection{Human Smooth Muscle Cells on the Silk Fibroin-Modified PHBHHx}

4.2.1. Cells Vitality. The results of MTT assay for the human smooth muscle cells (hSMCs) on the porous scaffolds are 


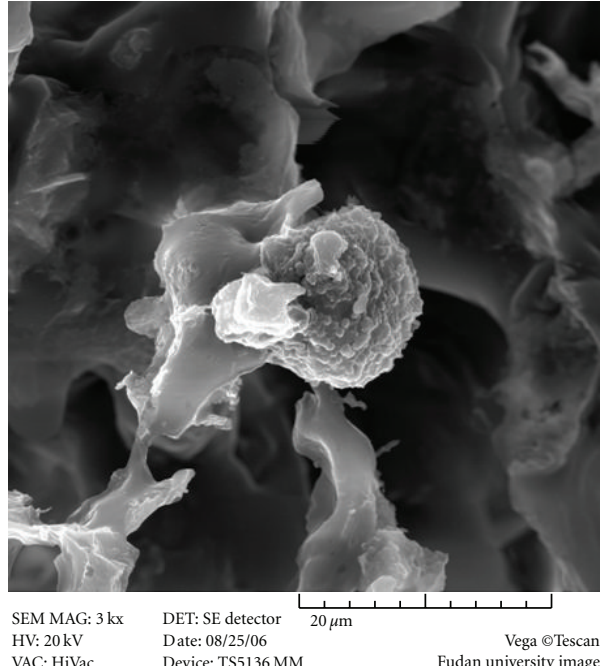

(a)

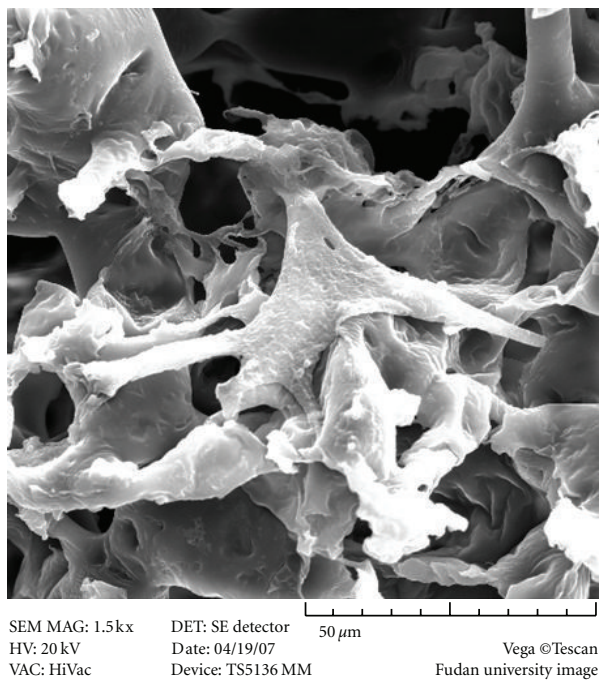

(c)

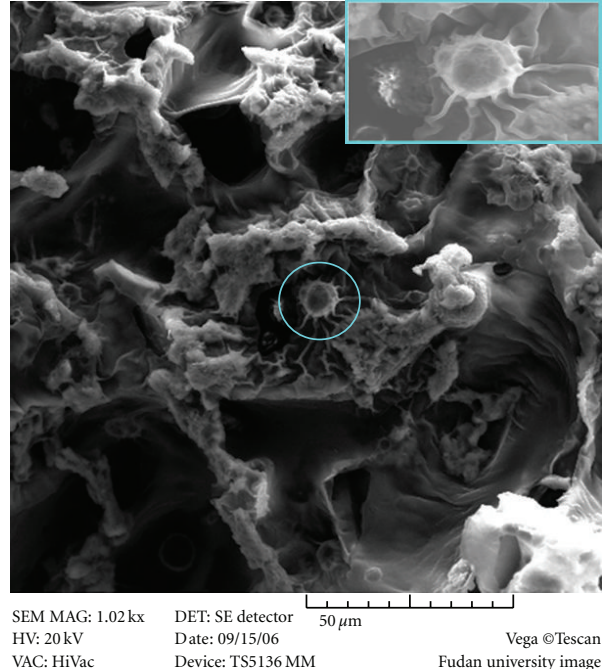

(b)

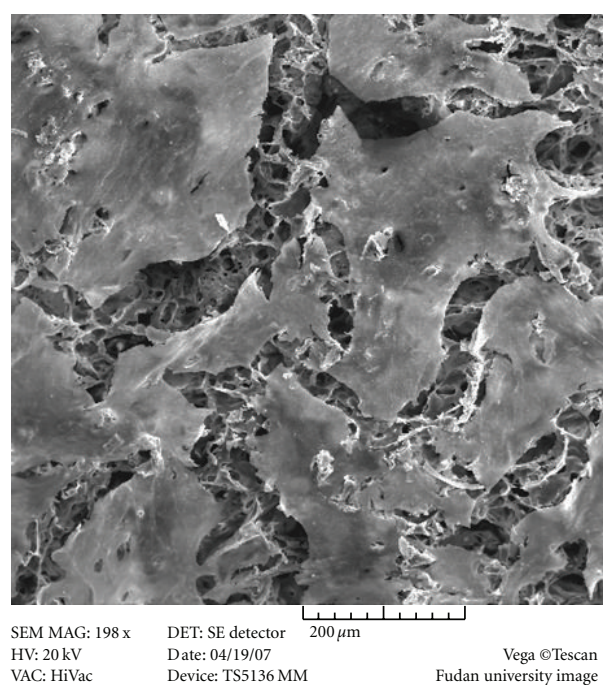

(d)

FiguRE 10: SEM images of the morphologies of human smooth muscle cells (hSMCs) on the silk fibroin-modified PHBHHx scaffolds. (a) 1 day of culture; (b) and (c) 3 days of culture; (d) 7 days of culture. The circulated image is magnified cell shown at the top right corner of (b) $[25]$.

shown in Figure 9. It can be found that the SF-modified PHBHHx porous scaffolds have obvious advantage for supporting the hSMCs growth and proliferation in the culture days between 3 to 21 days. In particular, in two weeks culture, the hSMCs on the unmodified scaffold nearly cease proliferating while they continue proliferating on the SFmodified scaffold.

4.2.2. Cells Morphology. The morphologies of hSMCs cultured in silk fibroin-modified porous PHBHHx scaffold are also observed by SEM as shown in Figure 10. From 1 to 7 days of culture, hSMCs show the round cells after 1 day of culture (Figure 10(a)), and then the cells stretch out as the pseudopodia (see inserted illustration in Figure 10(b)) after 3 days culture, and some cells migrate into the pores (Figure 10(c)) and form the cell monolayer on the surface of scaffolds in 7 days of culture (Figure 10(d)).
The SF-modified PHBHHx porous scaffold is verified to better support the hSMCs growth and proliferation than the unmodified one, evaluated by the methods of MTT assay and SEM observation.

\subsection{Human Umbilical Vein Endothelial Cells and ECV304} on the Silk Fibroin-Modified PHBHHx. Endothelial cells as a component of inner surface in the blood vessel directly contact with the blood and functionalize as an anti-blood clotting layer. Herein, human umbilical vein endothelial cells (HUVECs) are isolated from the human umbilical vein. The ECV304 is a kind of endothelial-like cell line.

4.3.1. Cells Vitality. Figure 11 shows the results of MTT assay of HUVECs and ECV304 cultured on the porous PHBHHx scaffold. After 1 day of culture, HUVECs (Figure 11(a)) and ECV304 (Figure 11(b)) on the SF-modified scaffolds show 


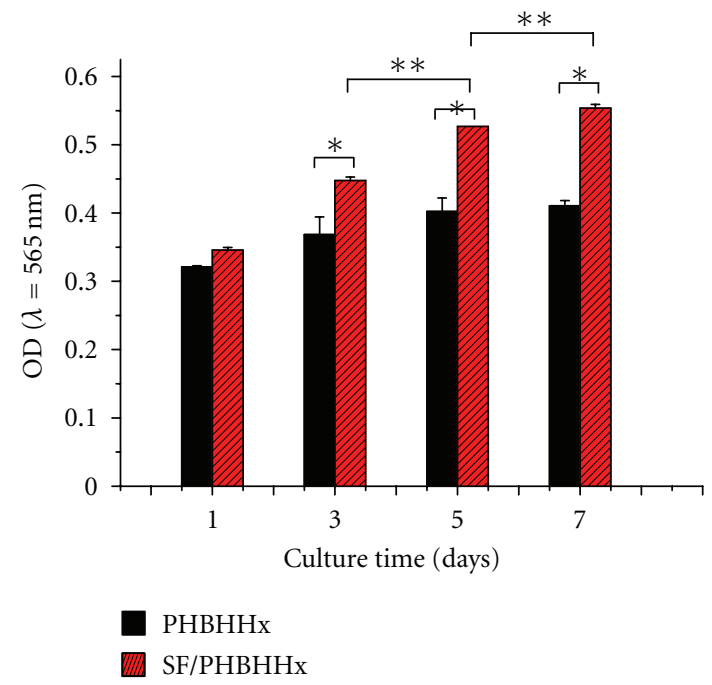

(a)

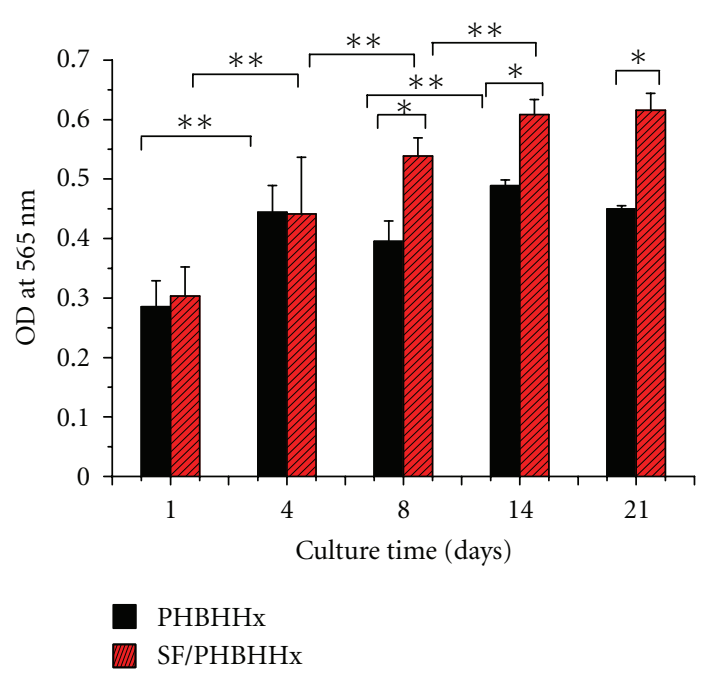

(b)

Figure 11: MTT assay of HUVECs (a) [28] and ECV304 (b) [20] cultured on the SF-modified PHBHHx and PHBHHx scaffolds. * comparison of cell activity on SF/PHBHHx and PHBHHx scaffolds at the same culture time $(P<0.05)$; ** comparison of cell activity on the same kind of scaffolds at different culture time $(P<0.01)$.

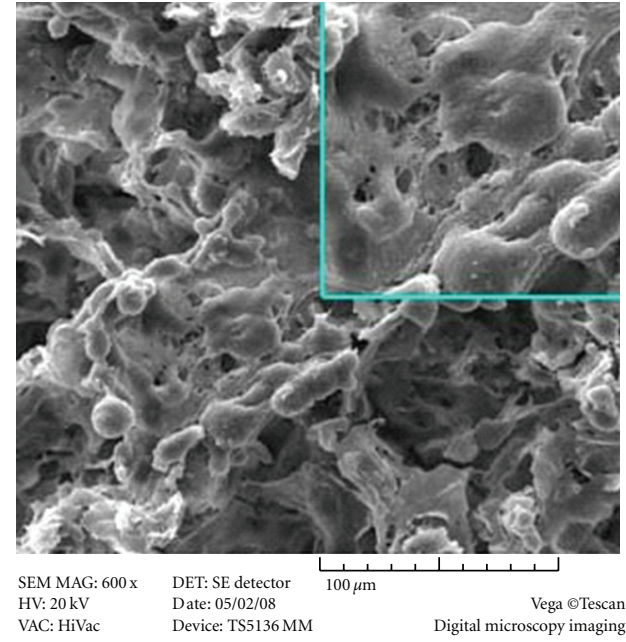

(a)

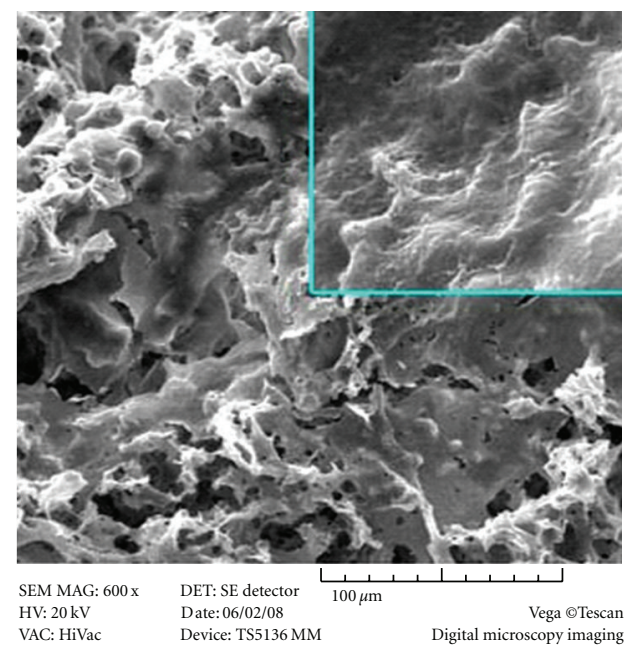

(c)

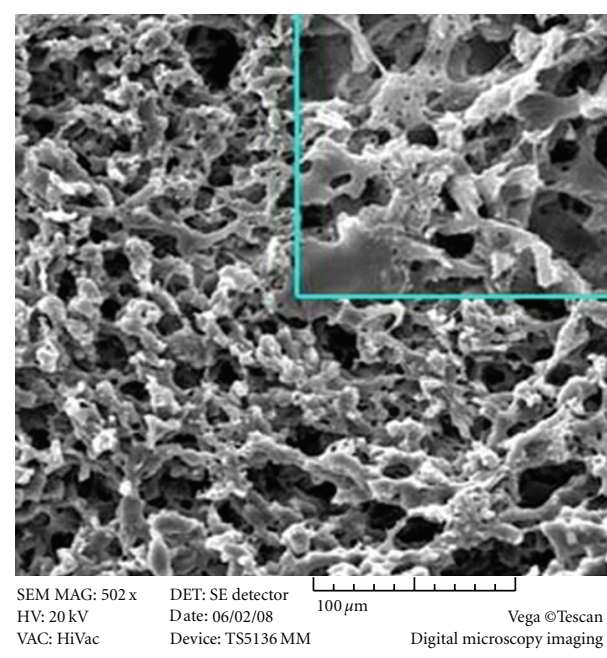

(b)

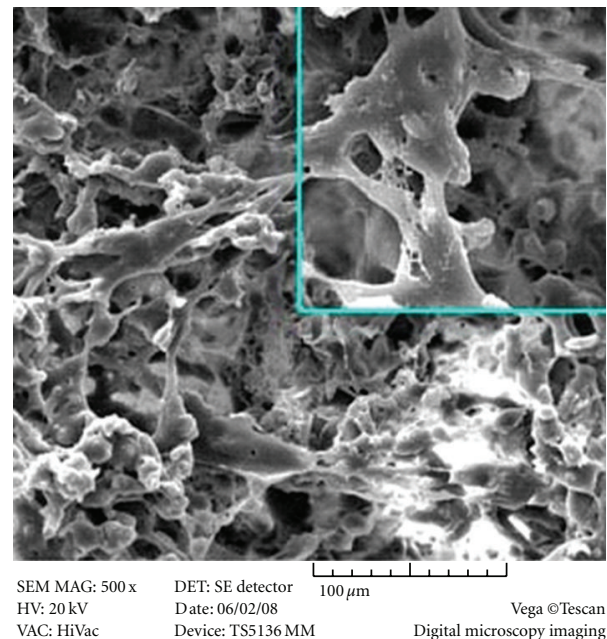

(d)

FigURE 12: SEM images of HUVECs morphologies in the scaffolds at different culture times. (a) and (c) show the cells cultured for 3, 5 days on the SF-modified PHBHHx scaffolds, respectively; (b) and (d) show the cells cultured for 3, 5 days respectively, in the PHBHHx porous scaffolds. The magnified images are at the top right corner of each image [28]. 


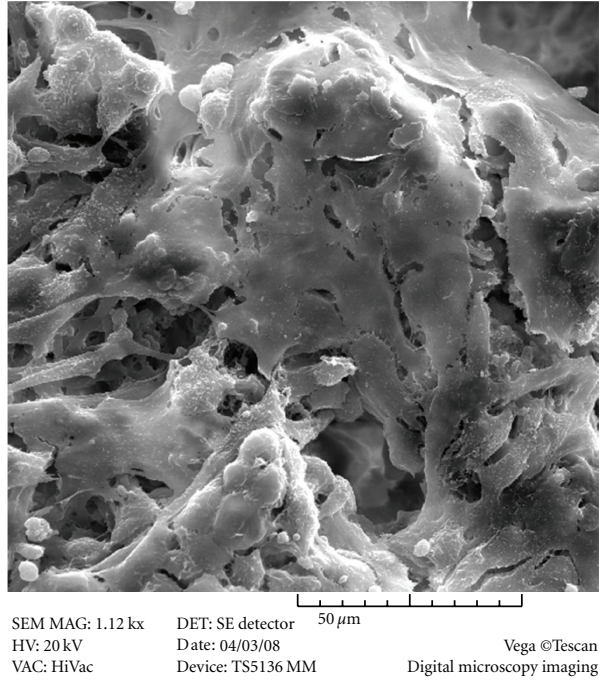

(a)

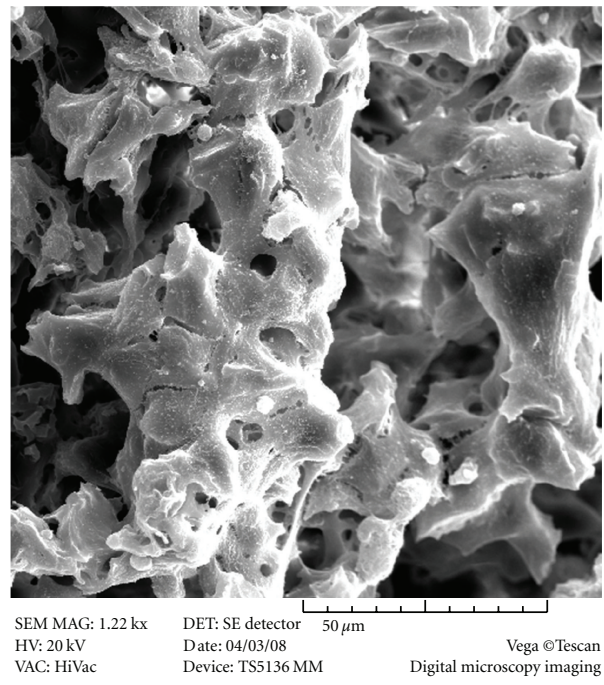

(b)

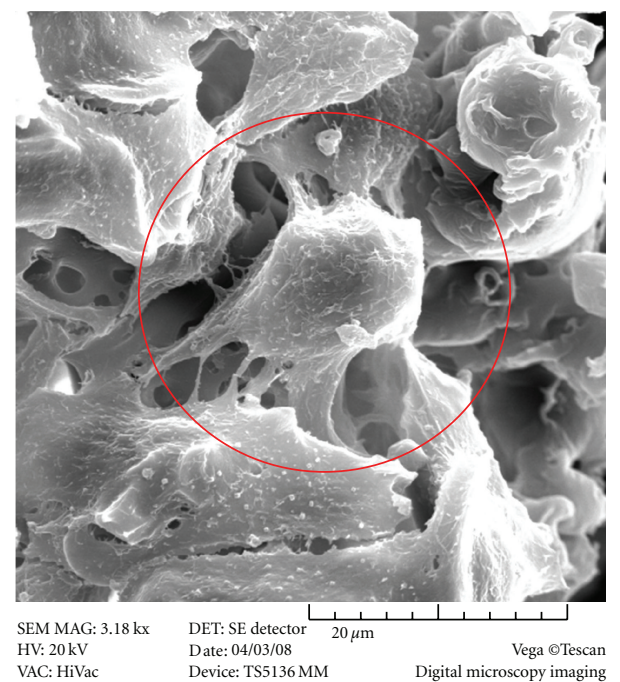

(c)

Figure 13: SEM images of ECV304 on the 3D porous SF-modified PHBHHx (a) and PHBHHx (b) scaffolds for 7 days of culture as well as an amplified single cell on the 3D porous PHBHHx scaffold for 7 days of culture (c) [20].

the better vitality than that on the unmodified scaffolds. After 3,5 , and 7 days of culture, there are obvious differences between two kinds of scaffolds for the cells. The SF-modified scaffolds have better biocompatibility to support the HUVECs and ECV304 growth and proliferation.

4.3.2. Cells Morphology. The morphologies of HUVEC are shown in Figure 12. After 3 days of culture, the HUVECs on SF-modified porous PHBHHx scaffold show better vitality (Figure 12(a)) than that on unmodified scaffold (Figure 12(b)), and then, after 5 days of culture, continuous cell monolayer is formed on modified scaffold (Figure 12(c)), which cannot be formed on the unmodified one (Figure 12(d)).

The ECV304 cells on the SF-modified PHBHHx scaffolds form a continuous monolayer with closer connection (Figure 13(a)) than those on the PHBHHx scaffold (Figure 13(b)) after 7 days of culture. Moreover, the cells grew more evenly on the SF-modified PHBHHx porous scaffolds and spread with close space between each other. Figure 13(c) shows an amplified single cell image where the cell grows well and stretches out into the pores of the PHBHHx scaffold after 7 days of culture. The pseudopodium and microvillus are clearly observed in the image. It indicates that there is a proper environment for the cells growth on the SF-modified PHBHHx scaffold.

4.3.3. Extracellular Matrix Secretion from HUVECs. Extracellular matrix (ECM) is the secretion of the cells during the growth and proliferation of the cells in vitro and in vivo. The collagen is one of the important components in the ECM. The secretion function of the cells cultured on the scaffolds can be evaluated by the content of the collagen. The trend of the collagen secretion from the HUVECs cultured on the porous scaffold within 5 to 15 days is shown in Figure 14. We find that the collagen content on the SF-modified scaffold is significantly higher than that on the unmodified scaffold. 


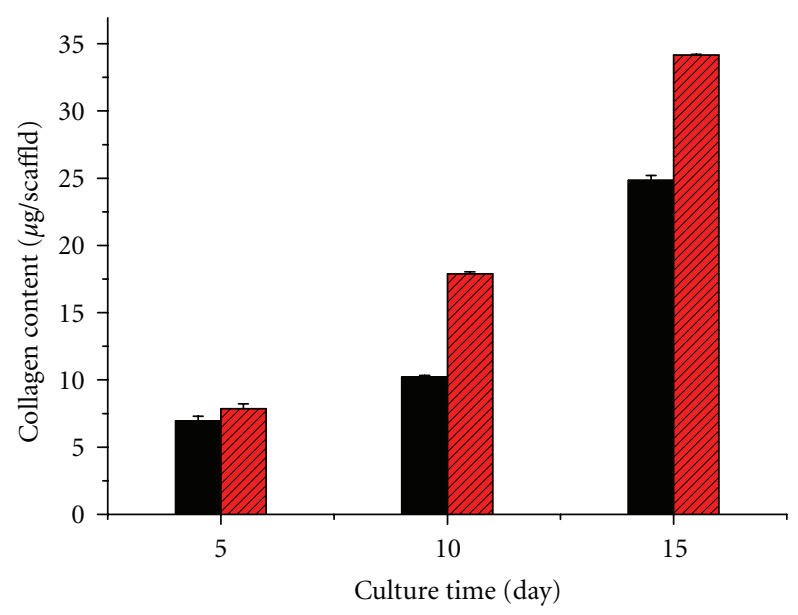

PHBHHx

SF/PHBHHx

FIGURE 14: The secreted collagen content from HUVECs on SF/ PHBHHx and PHBHHx scaffolds for different cells culture time [28].

The conclusion can be made that SF modification benefits the ECM secretion of the cells and the formation of the cell network in the porous scaffolds.

\section{Conclusion}

To achieve an ideal biodegradable polymer implant with less inflammation reactions, antithrombin, and proper hydrophilic surface for the clinical applications, a natural protein, silk fibroin, which possesses excellent biocompatibility, is utilized as a modificator to treat the PHBHHx porous scaffolds. The mechanical tests show that the surface modification by $0.75 \mathrm{wt} \%$ concentration of the silk fibroin does not affect the mechanical property of $\mathrm{PHBHHx}$ material and meets the requirement of the cardiovascular tissue engineering. The result of water diffusion in the $\mathrm{PHBHHx}(\mathrm{HHx}=$ $12 \mathrm{~mol} \%$ ) indicates that the higher water diffusion coefficient of PHBHHx than that of PHB implies that the PHBHHx is more biocompatible, especially for the transportation of water and the soluble nutrition into the cells through the polymer scaffold. ATR-FTIR results prove that the silk fibroin can adhere on the PHBHHx surface firmly, even flushed by the PBS for 21 days. Three types of cardiovascularrelated cells, such as human fibroblasts, smooth muscle cells, human umbilical vascular endothelial cells, and endotheliallike cell line-ECV304, were cultured on the SF-modified PHBHHx porous scaffolds demonstrating that SF-modified PHBHHx porous scaffolds have a better cell adhesion and proliferation than PHBHHx. As a result, SF-modified $\mathrm{PHBHHx}$ porous scaffolds have potential application into the tissue-engineered cardiovascular materials.

\section{Acknowledgment}

This project was supported by the Natural Science Foundation of China (nos. 10475017, 20673022, 21074025).

\section{References}

[1] I. Vesely, "Heart valve tissue engineering," Circulation Research, vol. 97, no. 8, pp. 743-755, 2005.

[2] R. Langer and J. P. Vacanti, “Tissue engineering," Science, vol. 260, no. 5110, pp. 920-926, 1993.

[3] S. Y. Lee, "Bacterial polyhydroxyalkanoates," Biotechnology and Bioengineering, vol. 49, no. 1, pp. 1-14, 1996.

[4] X. Hu, H. Shen, F. Yang, J. Bei, and S. Wang, "Preparation and cell affinity of microtubular orientation-structured PLGA(70/30) blood vessel scaffold," Biomaterials, vol. 29, no. 21, pp. 3128-3136, 2008.

[5] P. Sangsanoh, S. Waleetorncheepsawat, O. Suwantong et al., "In vitro biocompatability of Schwann cells on surfaces of biocompatible polymeric electrospun fibrous and solution-cast film scaffolds," Biomacromolecules, vol. 8, no. 5, pp. 15871594, 2007.

[6] N. Mei, G. Chen, P. Zhou et al., "Biocompatibility of poly( $(\varepsilon-$ caprolactone) scaffold modified by chitosan-the fibroblasts proliferation in vitro," Journal of Biomaterials Applications, vol. 19, no. 4, pp. 323-339, 2005.

[7] G. Q. Chen and Q. Wu, "The application of polyhydroxyalkanoates as tissue engineering materials," Biomaterials, vol. 26, no. 33, pp. 6565-6578, 2005.

[8] S. Cheng, Q. Wu, F. Yang, M. Xu, M. Leski, and G. Q. Chen, "Influence of DL- $\beta$-hydroxybutyric acid on cell proliferation and calcium influx," Biomacromolecules, vol. 6, no. 2, pp. 593597, 2005.

[9] Y. Doi, S. Kitamura, and H. Abe, "Microbial synthesis and characterization of poly(3-hydroxybutyrate-co-3-hydroxyhexanoate)," Macromolecules, vol. 28, no. 14, pp. 4822-4828, 1995.

[10] K. Zhao, Y. Deng, J. C. Chen, and G. Q. Chen, "Polyhydroxyalkanoate (PHA) scaffolds with good mechanical properties and biocompatibility," Biomaterials, vol. 24, no. 6, pp. 10411045, 2003.

[11] R. Sodian, S. P. Hoerstrup, J. S. Sperling et al., "Early in vivo experience with tissue-engineered trileaflet heart valves," Circulation, vol. 102, no. 19, pp. III22-III29, 2000.

[12] Y. W. Wang, Q. Wu, and G. Q. Chen, "Attachment, proliferation and differentiation of osteoblasts on random biopolyester poly(3-hydroxybutyrate-co-3-hydroxyhexanoate) scaffolds," Biomaterials, vol. 25, no. 4, pp. 669-675, 2004.

[13] X. H. Qu, Q. Wu, K. Y. Zhang, and G. Q. Chen, "In vivo studies of poly(3-hydroxybutyrate-co-3-hydroxyhexanoate) based polymers: biodegradation and tissue reactions," Biomaterials, vol. 27, no. 19, pp. 3540-3548, 2006.

[14] M. H. Ho, D. M. Wang, L. T. Hou, C. Y. Tu, H. J. Hsieh, and J. Y. Lai, "RGD immobilization on PLLA membranes to promote biocompatibility," Desalination, vol. 200, no. 1-3, pp. 501-502, 2006.

[15] Y. Wang, H. J. Kim, G. Vunjak-Novakovic, and D. L. Kaplan, "Stem cell-based tissue engineering with silk biomaterials," Biomaterials, vol. 27, no. 36, pp. 6064-6082, 2006.

[16] B. Panilaitis, G. H. Altman, J. Chen, H. J. Jin, V. Karageorgiou, and D. L. Kaplan, "Macrophage responses to silk," Biomaterials, vol. 24, no. 18, pp. 3079-3085, 2003.

[17] G. Chen, P. Zhou, N. Mei et al., "Silk fibroin modified porous poly ( $\varepsilon$-caprolactone) scaffold for human fibroblast culture in vitro," Journal of Materials Science: Materials in Medicine, vol. 15, no. 6, pp. 671-677, 2004.

[18] G. Chen, P. Zhou, L. F. Pan et al., "Growth of human fetal lung fibroblasts on the natural biomaterial-chitosan scaffold," Acta Chimica Sinica, vol. 62, no. 10, pp. 992-997, 2004. 
[19] N. Mei, P. Zhou, L. F. Pan et al., "Biocompatibility of poly (3-hydroxybutyrate-co-3-hydroxyhexanoate) modified by silk fibroin," Journal of Materials Science: Materials in Medicine, vol. 17, no. 8, pp. 749-758, 2006.

[20] M. Sun, L. F. Pan, S. Liu, and H. X. Yang, "Enhanced cell affinity of the silk fibroin modified PHBHHx material," Journal of Materials Science: Materials in Medicine, vol. 20, no. 8, pp. 1743-1751, 2009.

[21] H. X. Yang, M. Sun, and P. Zhou, "Investigation of water diffusion in poly(3-hydroxybutyrate-co-3-hydroxyhexanoate) by generalized two-dimensional correlation ATR-FTIR spectroscopy," Polymer, vol. 50, no. 6, pp. 1533-1540, 2009.

[22] A. L. Iordanskii and P. P. Kamaev, "The effect of preparation procedure on water sorption in membranes based on bacterial poly(3-hydroxybutyrate)," Vysokomolekularnye Soedineniya $A \mho B$, vol. 41, no. 2, pp. 374-378, 1999.

[23] A. L. Iordanskii, P. P. Kamaev, and G. E. Zaikov, "Water sorption and diffusion in poly-(3-hydroxybutyrate) films," Polymer-Plastics Technology and Engineering, vol. 38, no. 4, pp. 729-738, 1999.

[24] K. Cai, K. Yao, Y. Cui et al., "Influence of different surface modification treatments on poly(D,L-lactic acid) with silk fibroin and their effects on the culture of osteoblast in vitro," Biomaterials, vol. 23, no. 7, pp. 1603-1611, 2002.

[25] Y. Zhang, P. Zhou, L. F. Pan, S. Z. Xie, M. Sun, and W. T. Li, "Growth of human smooth muscle cells on the silk fibroin modified-polyhydroxyalkanoate scaffold," Acta Chimica Sinica, vol. 65, pp. 2935-2940, 2007.

[26] A. Padermshoke, H. Sato, Y. Katsumoto, S. Ekgasit, I. Noda, and Y. Ozaki, "Crystallization behavior of poly(3-hydroxybutyrate-co-3-hydroxyhexanoate) studied by 2D IR correlation spectroscopy," Polymer, vol. 45, no. 21, pp. 7159-7165, 2004.

[27] Y. W. Wang, Q. Wu, and G. Q. Chen, "Reduced mouse fibroblast cell growth by increased hydrophilicity of microbial polyhydroxyalkanoates via hyaluronan coating," Biomaterials, vol. 24, no. 25, pp. 4621-4629, 2003.

[28] M. Sun, H. X. Yang, P. Zhou, L. F. Pan, and S. Liu, "Biocompatibility of PHBHHx and silk fibroin-modified PHBHHx scaffolds with human umbilical vein endothelial cells," Acta Polymerica Sinica, vol. 0, no. 6, pp. 1430-1436, 2010. 

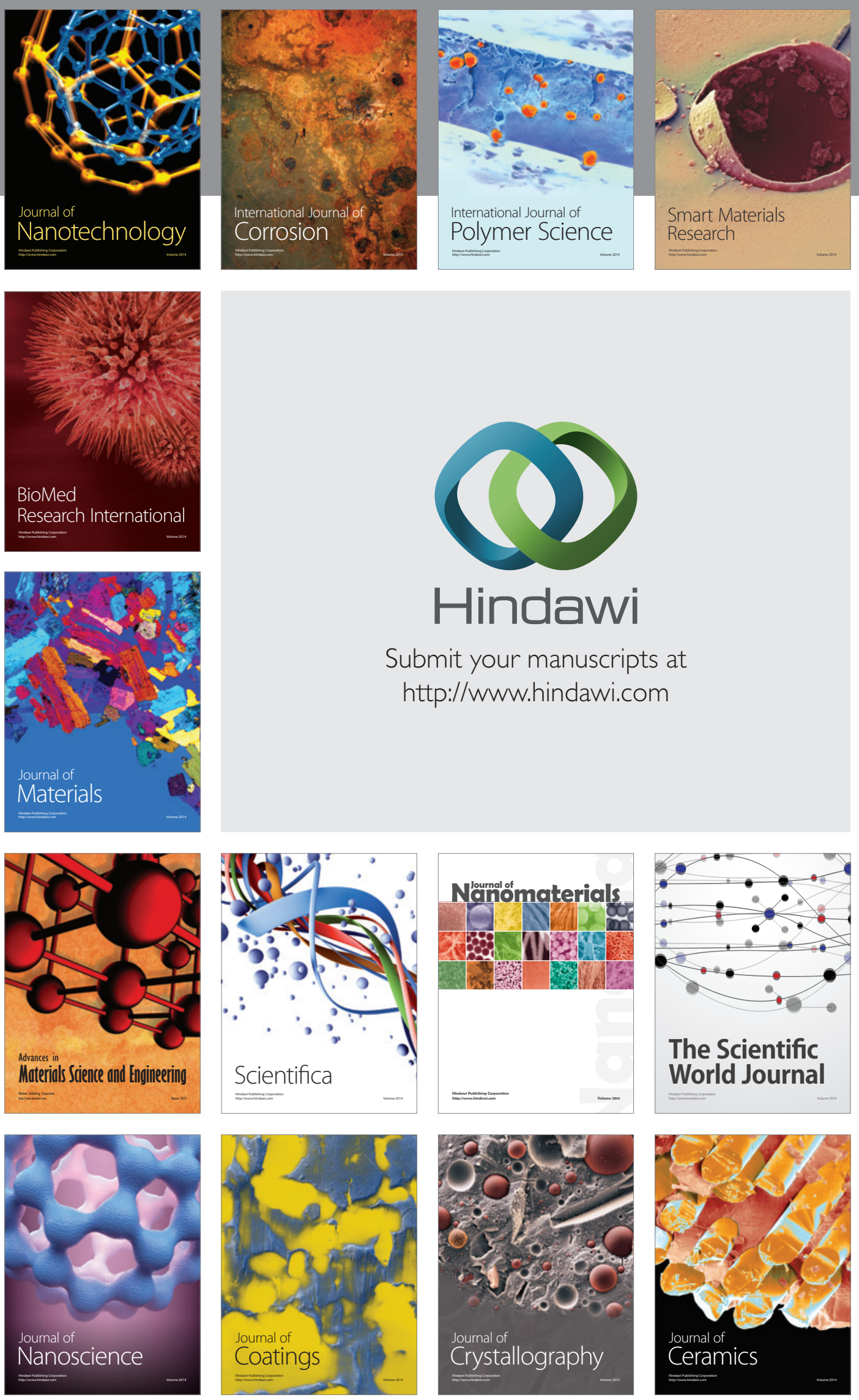

The Scientific World Journal

Submit your manuscripts at

http://www.hindawi.com

\section{World Journal}

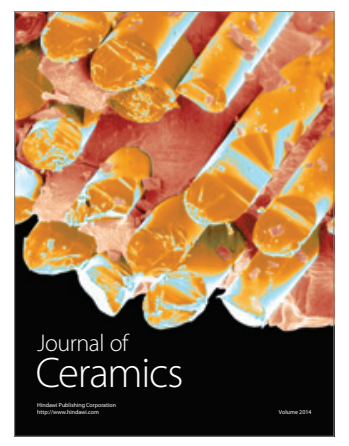

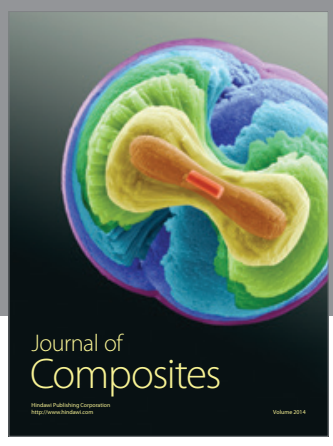
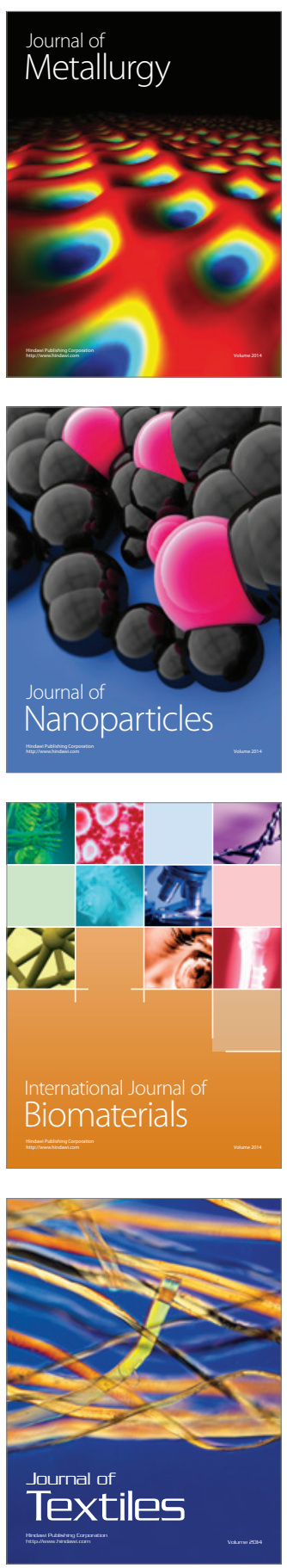